\title{
Interactive Exploration of Left Atrium Population- Level Morphology in Atrial Fibrillation Patients
}

\author{
Tim Sodergren $^{1}$, Anupama Goparaju $^{1}$, Alan Morris ${ }^{2}$, Evgueni Kholmovski ${ }^{2,3}$, \\ Nassir Marrouche ${ }^{2}$, Joshua Cates ${ }^{2}$, Shireen Elhabian ${ }^{1}$ \\ ${ }^{1}$ Scientific Computing and Imaging Institute, School of Computing, \\ University of Utah, Salt Lake City, Utah, USA \\ ${ }^{2}$ Comprehensive Arrhythmia Research and Management Center, Division of Cardiovascular \\ Medicine, School of Medicine, University of Utah, Salt Lake City, Utah, USA \\ ${ }^{3}$ Department of Radiology and Imaging Sciences, School of Medicine, \\ University of Utah, Salt Lake City, Utah, USA
}

\begin{abstract}
We have developed computational methods for interactively exploring the shape of the left-atrium in a population of atrial fibrillation patients. We analyze the LA shape through a shape-learning algorithm termed as particlebased modeling (PBM), in which we extract surface contours from a population of images and then parameterize population-level shape statistics through the automatic placement of a dense set of homologous landmark positions (aka correspondences) using an optimization on information content. We then generate a 2-D embedding of the resulting high-dimensional dataset which allows us to visualize the data on a scatter plot, with each data point representing a single sample. This parameterization of the shape characteristics of samples collapsed onto a single plot gives us a visual representation of the population-level morphology of the data. Cardiac MR angiography data from 212 AF patients was collected retrospectively from a database of AF patients at the University of Utah. From the 2-D scatter plot, we were able to interactively select individual samples, view their shapes, and see associated clinical data. We can also map new patients to infer their relations to other patients in the population via querying nearby samples and viewing their clinical data.
\end{abstract}

\section{Introduction}

It has been hypothesized that left atrium (LA) shape may be an indicator of atrial fibrillation (AF) and plays a role in thrombus formation. This study aims at developing computational methods for interactive visualization of 3D LA shapes utilizing outputs from shape-driven statistical models to allow a clinician to make inferences about a patient in reference to an entire population.

\subsection{Particle-Based Modeling}

Because of the complex nature of cardiac anatomy, explicit parameterizations are limited in their ability to fully describe the shape of a given sample. For example, a spherical representation of the LA can only represent surfaces with spherical topologies whereas the actual shape will be far more complex. Particle-based modeling (PBM) instead seeks to represent shapes as a set of points constrained to lie on an implicit surface, in this case the segmented left atrial volume. See the references for a more detailed explanation of PBM [1-4]. PBM represents a population of shapes as a dense set of homologous landmarks termed correspondence positions . These landmarks iteratively reposition themselves through an optimization algorithm. This optimization seeks minimize the entropy of the model which is a metric of information content. The model is also able to capture areas of higher detail by increasing sampling rates in regions of higher surface curvature (Figure 1).

\subsection{2-D Embedding}

While the result of PBM provides a detailed description of a population of shapes, the resulting parameterization is high dimensional, on the order of hundreds to several thousand dimensions depending on the number of correspondences. This can visualizing a dataset challenging. However, while the dimensionality of the dataset may be very high, the intrinsic dimension of the data is often much smaller. It is useful then, to employ some dimension reduction techiques to limit the size of the dataset to something more manageable particularly for visualizing a 


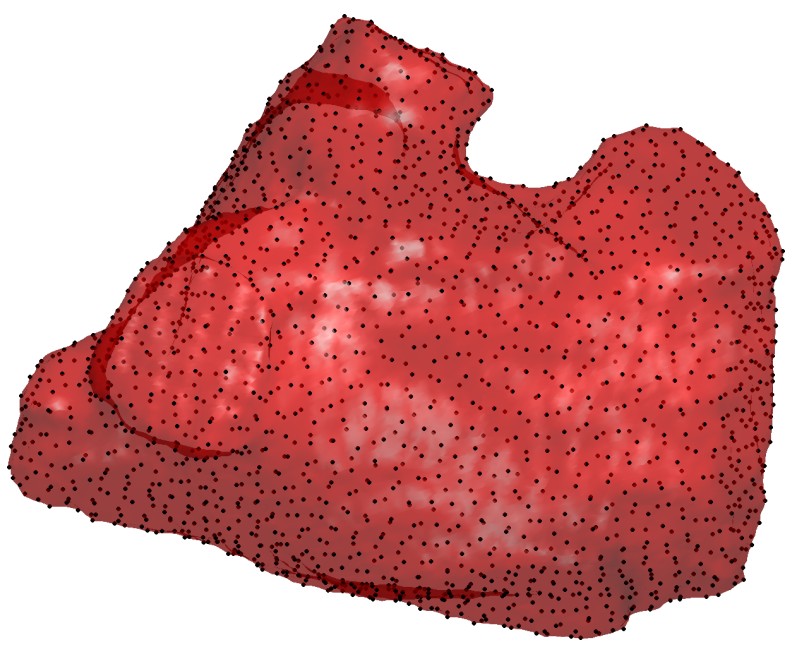

Figure 1. Example of PBM representation of a sample segmented left-atrium. Each point represents a landmark position that corresponds to the same point across the entire population of samples.

high dimensional dataset. Principal component analysis (PCA) is by far the most commonly used dimension reduction technique but is limited in it's ability to describe complex datasets, particular those with non-linear relationships as it relies on linear relationships between dimensions. We instead employ a technique called t-distributed stochastic neighbor embedding (t-SNE) [5]. T-SNE is a non-linear dimensionality reduction method for embedding high-dimensional data into a two or three dimensional space for visualization by grouping points in such a way that similar objects are near each other and dissimilar objects are distant with high probability. It works by defining each point in high-dimensional space as a probability distribution and mapping those probability distributions to the low dimensional space.

Having this 2-D representation then allows a clinician to explore a population of LA shapes to identify trends and similarities and then compare this information with sample level metadata such as outcomes or demographic.

\section{Results}

We collected 212 samples of cardiac MR angiography data from a database of AF patients from the University of Utah. For each sample, we also collected associated demographics and clinical information such as AF type and recurrence. After segmenting the LA volume, we constructed PBM shape models for the LA endocardial surface using 2048 correspondence points per sample. We then constructed a 2-D representation of the data via t-SNE as shown in Figure 2. This is a visualization of the distribution of shape parameters for our population. It should be noted that the actual magnitudes of the axes of this plot have no physical meaning. They are instead a representation of the relative differences between shapes. The distance between any two points in this collapsed subspace can be thought of as a proxy for the dissimilarity of the LA shapes associated with each.

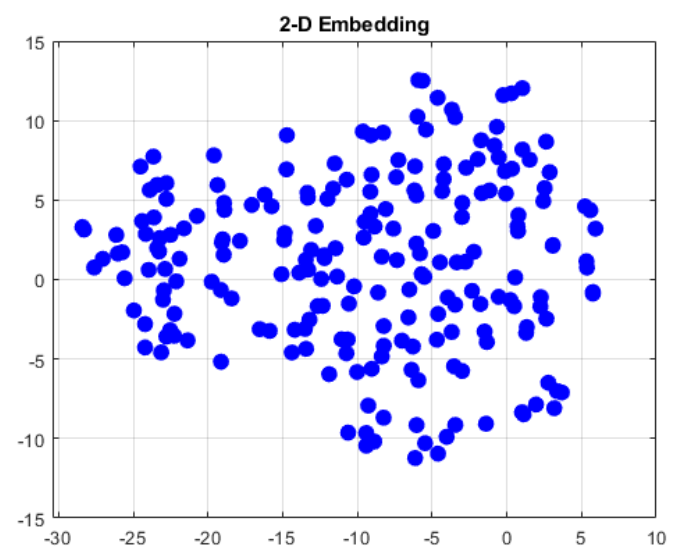

Figure 2. Two-dimensional representation of LA population via t-SNE. Each point represents a single patient and all points are distributed such that their proximity is directly related to their proximity in the high-dimensional shape space. Points close to each other have similar shapes while distant point will have dissimilar shapes.

We then used this 2-D model, along with the highdimensional individual PBM models and associated data to construct an interactive visualization tool for exploring the LA population. Individual shapes, such as in Figure 1 , and their associated clinical data can be displayed by selecting it's associated point in the 2-D plot. This also allows for the visualization of the distribution of properties such as atrial fibrillation type (Figure 3).

Additionally, we can query the shape space by comparing new shapes against this population by comparing nearby points, their shapes and clinical data to make inferences about the new sample. We illustrate this capability in Figure 4. If we treat the sample highlighted in green as a new sample, we can visualize the LA shape of the nearest samples from the database and generate a table of clinical data from this subpopulation.

\begin{tabular}{|c|c|c|c|c|c|c|}
\hline ID & Gender & Age & AF Type & Thrombus & Stroke & Recurrence \\
\hline \hline 43 & F & 79.6 & persistent & no & no & no \\
\hline \hline 184 & F & 61.7 & persistent & no & no & no \\
\hline \hline 12 & M & 68.5 & persistent & no & no & no \\
\hline
\end{tabular}

\section{Conclusions}

Through PBM and dimensionality reduction, we were able to represent the population-level LA shape character- 


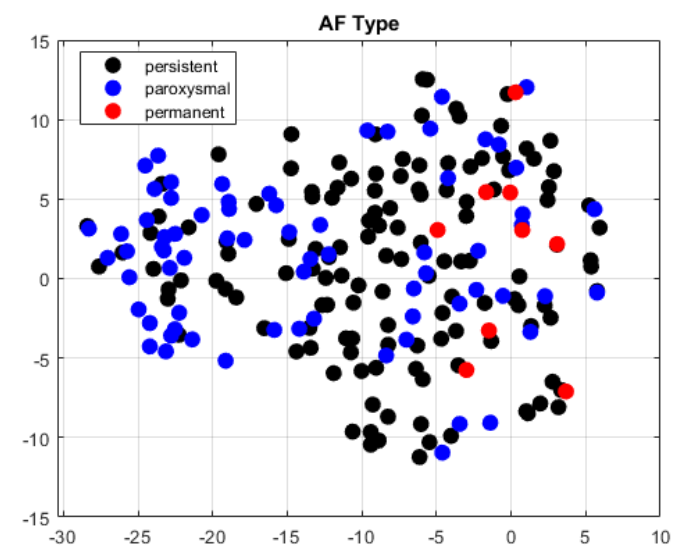

Figure 3. Distribution of AF type in LA population in the low-dimensional subspace.

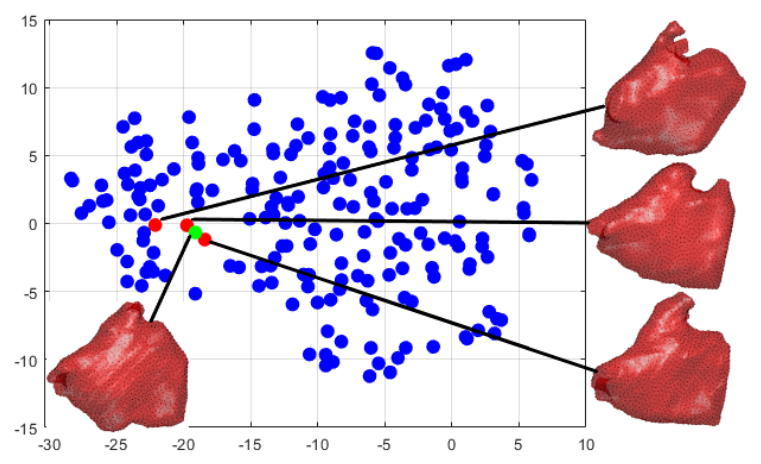

Figure 4. caption

istics, make comparisons within a population, and make comparisons of new shapes to an existing population, which may allow a clinician to make inferences about clinical outcomes, reveal population-level trends, and facilitate shape-driven hypothesis generation.

\section{Acknowledgements}

\section{References}

[1] Oguz I, Cates J, Datar M, Paniagua B, Fletcher T, Vachet C, Styner M, Whitaker R. Entropy-based particle correspondence for shape populations. Int J Comput Assist Radiol Surg Jul 2016;11(7):1221-1232. ISSN 1861-6410.

[2] Cates J, Fletcher PT, Styner M, Shenton M, Whitaker R. Shape modeling and analysis with entropy-based particle systems. Inf Process Med Imaging 2007;20:333-345. ISSN 1011-2499.

[3] Cates J, Fletcher PT, Styner M, Hazlett HC, Whitaker R. Particle-based shape analysis of multi-object complexes. Med Image Comput Comput Assist Interv 2008; 11(Pt 1):477-485.

[4] Datar M, Gur Y, Paniagua B, Styner M, Whitaker R. Geometric correspondence for ensembles of nonregular shapes. Med Image Comput Comput Assist Interv 2011;14(Pt 2):368375.

[5] van der Maaten L, Hinton G. Visualizing data using t-SNE. Journal of Machine Learning Research 2008;9:2579-2605.

\section{Address for correspondence:}

Tim Sodergren

72 Central Campus Drive, 3rd floor desk, Salt Lake City, Utah84112, USA

tsodergren@sci.utah.edu 